\title{
Conséquences des sécheresses 1985 et 1986 dans le Sud-Ouest de la France : Elaboration d'un programme de lutte
}

\author{
J.Y. Ollivier \\ Ministère de l'Agriculture et de la Forêt, Paris
}

\begin{abstract}
Les années 1985 et 1986 ont été marquées dans le SudOuest par une "sécheresse inhabituelle», qui a eu un impact important sur l'économie agricole de ces régions. Ces circonstances ont mis en lumière l'importance de l'irrigation comme facteur de modernisation des exploitations agricoles et la nécessité de renforcer les ressources en eau de ces régions.
\end{abstract}

\section{Caractéristiques de la sécheresse de 1985 et 1986 dans le Sud-Ouest}

D'une façon générale, pour parler de « sécheresse inhabituelle", il faut qu'une période suffisamment longue connaisse, sur une aire géographique suffisamment étendue, des précipitations nettement inférieures à la moyenne.

Une analyse de la pluviométrie des années 1985 et 1986 dans le Sud-Ouest permet de constater une sécheresse exceptionnelle concernant en particulier l'été et l'automne de ces deux années.
Le tableau ci-dessous indique le rapport des précipitations mensuelles à la normale, calculé sur l'ensemble des six stations suivantes (Bordeaux - Mont-de-Marsan - Agen - Gourdon - Toulouse - Carcassonne).

\begin{tabular}{rrrrrrrrrrrrr} 
& \multicolumn{1}{c}{ J } & \multicolumn{1}{c}{ F } & \multicolumn{1}{c}{ M } & \multicolumn{1}{c}{ A } & \multicolumn{1}{c}{ M } & \multicolumn{1}{c}{ J } & J & A & S & 0 & N & D \\
1985 & 78 & 109 & 143 & 109 & 148 & 106 & $\underline{84}$ & $\underline{42}$ & $\underline{2}$ & $\underline{54}$ & $\underline{69}$ & 73 \\
1986 & 197 & 92 & 80 & 186 & $\underline{55}$ & $\underline{24}$ & $\underline{33}$ & $\underline{57}$ & 97 & 69 & 65 & 96 \\
1987 & 38 & 81 & 105 & 84 & 41 & 112 & 163 & & & & &
\end{tabular}

Après une première moitié de 1985 généralement plus arrosée que d'habitude, les précipitations de chaque mois de juillet à décembre sont nettement inférieures à la normale : il s'agit d'une sécheresse d'été-automne.

En considérant les trois mois d'été (juillet à septembre), pour lesquels les conséquences agricoles sont les plus importantes, la carte numéro 1 montre l'étendue et l'intensité du phénomène: presque toute la France est

\section{Consequences of the droughts of 85 and 86 in the south-west of France. Setting up a combat programme}

The years 1985 and 86 were marked by two successive droughts in the south-west of France which had a big impact on the agricultural economy.

These circumstances highlighted the importance of irrigation as a factor in modernizing farms in these regions. The development of agricultural hydraulics has greatly increased and water resources, have been shown to be the limiting factor to irrigation.

A precise inventory, by sub-basins, was therefore drawn up to study the situation from the water resources/needs point of view, taking into account the prospect of increased irrigation.

From this work, a major building programme has been made part of the State-Region Contracts Plan. This will enable resources to be increased by approximately 150 million cubic meters for the following regions: Midi-Pyrénées, Aquitaine and Poitou-Charente. 
touchée, mais c'est du centre du Bassin Aquitain à l'Auvergne que le phénomène est le plus marqué ; il n'est tombé qu'un quart des précipitations habituelles, ce qui représente un déficit de $150 \mathrm{~mm}$ environ.

En 1986, les quatre premiers mois ont été globalement bien arrosés (surtout janvier et avril). Une nouvelle période sèche s'installe ensuite qui va durer de mai 1986 à mai 1987.

Pour la période de mai à septembre 1986, la carte de la figure 2 montre qu'il s'agit d'une sécheresse surtout marquée dans le Sud-Ouest et le Sud-Est, avec des précipitations comprises entre $40 \%$ et $50 \%$ de la normale, et des déficits par rapport à la normale encore voisins de $150 \mathrm{~mm}$.

La succession de ces deux années sèches a eu une répercussion importante sur l'économie des exploitations du Sud-Ouest (voir fig. I et 2).

\section{L'irrigation dans le Sud de la France : un enjeu impor- tant}

L'agriculture française est au cœur de la concurrence internationale et se trouve confrontée au contexte économique agricole général : crise économique - surproduction par rapport aux besoins des pays solvables, écoulement contrariés des productions agricoles, marges brutes diminuées...

Deux années de sécheresse successives ne peuvent donc qu'aggraver les difficultés d'exploitations agricoles non équipées pour compenser cette absence d'eau. Elles ont également mis en lumière le rôle important de l'irrigation pour moderniser l'agriculture de ces régions : elle procure, outre une assurance contre les années sèches, une meilleure capacité d'adaptation aux exploitations.

La maîtrise de l'eau permet aux agriculteurs d'améliorer ainsi leur productivité, de rester compétitifs et d'être à l'écoute des marchés et des possibilités de débouchés à l'intérieur comme à l'extérieur de la communauté.

L'irrigation favorise la diversification et l'adaptation aux marchés et contribue à l'organisation et à la consolidation des filières locales de production.

Ainsi, dans le Sud-Ouest, si l'activité agricole peut certes être pratiquée sans irrigation, les rendements sont ainsi médiocres et très irréguliers d'une année à l'autre.

L'introduction de l'irrigation rend ainsi possible, outre la régularité des rendements, une diversification des systèmes de production en permettant l'introduction de cultures plus rémunératrices mais exigeantes en eau: cultures de semences et d'oléoprotéagineux, amélioration des cultures de fruits et légumes.

Les apports moyens annuels sont de 1200 à $2000 \mathrm{~m}^{3} /$ ha.

Dans les régions méridionales, un taux annuel d'utilisation élevé des installations, la forte demande des agriculteurs et l'insuffisance fréquente des ressources en eau justifient un développement important des équipements collectifs publics constitué d'ouvrages fixes de stockage,

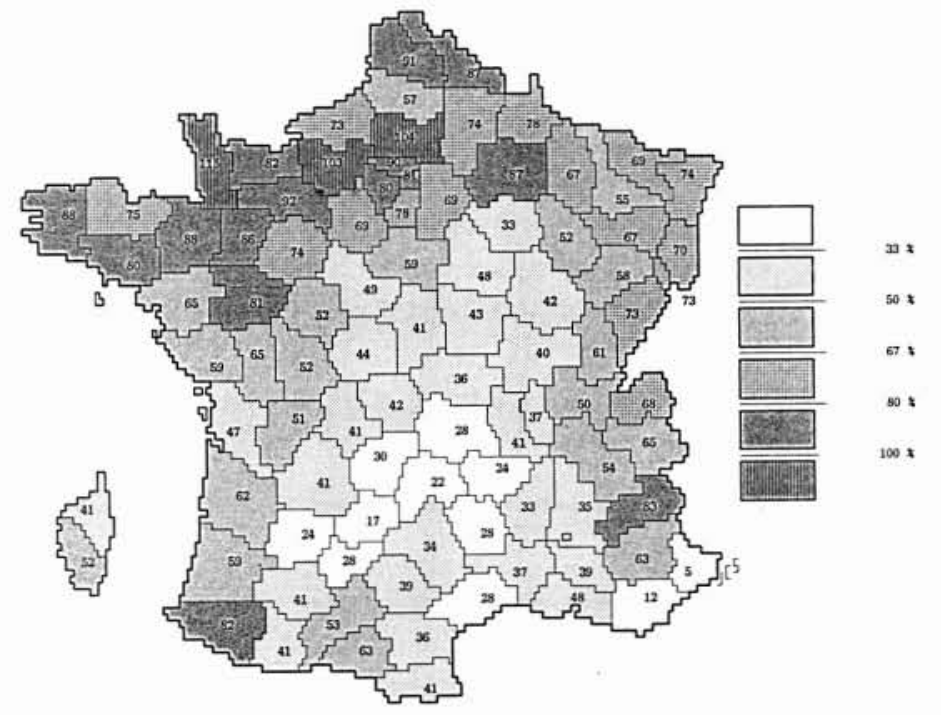

1. Précipitations de juillet-septembre 1985 (rapport à la normale).

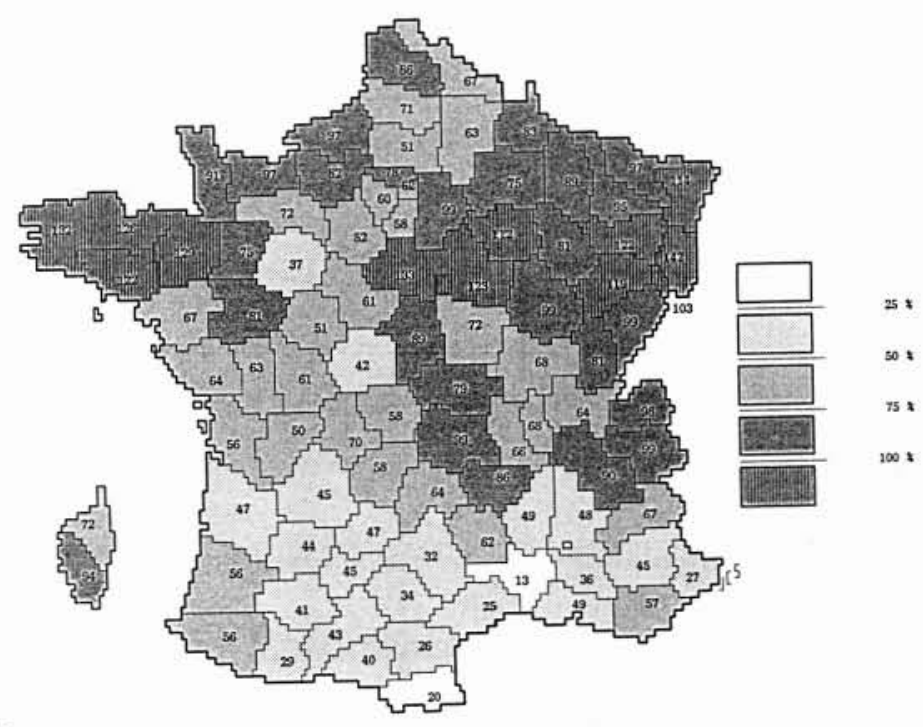

2. Précipitations de mai à août 1986 (rapport ả la normale).

transport et de distribution d'eau: les sociétés d'aménagement régional (Compagnie d'aménagement des Côteaux de Gascogne...) jouent un rôle important dans la création de ces équipements.

Bien évidemment, aucun projet d'investissement hydroagricole ne doit être réalisé sans une démarche préalable raisonnée, permettant d'intégrer la connaissance des milieux (données pédologiques, climatiques) celle de l'économie de l'exploitation ainsi que le projet technique et les perspectives des marchés agricoles de façon à pouvoir valider les investissements envisagés.

Cette prise de conscience par de nombreux agriculteurs que le facteur " maîtrise de l'eau » est un facteur important de modernisation a été fortement renforcée par les sécheresses consécutives des années 1985 et 1986.

Les ressources en eau sont clairement apparues comme le facteur limitant d'un développement raisonné de l'irrigation dans le Sud-Ouest. 


\section{La constitution des ressources en eau à usage agricole}

Dans le but de préparer les futurs contrats de Plan EtatRégions, qui seront mis en œuvre sur la période 1989-1993, un bilan des besoins en eau à usage agricole a été établi en 1987 pour le Sud-Ouest. L'objectif de l'étude était :

- de déterminer petits bassins par petits bassins (principalement 32 en Aquitaine, 33 en Midi-Pyrénées) dans quelle mesure les débits d'étiage des rivières permettraient de faire face aux besoins des agriculteurs en eau d'irrigation par pompage dans le lit ou dans la nappe alluviale sans que les autres usagers en souffrent, et ce dans l'immédiat puis dans une prévision à moyen terme (an 2000);

- en cas de débit insuffisant, de donner un ordre de grandeur du volume à stocker pour les besoins de l'agriculture.

Un premier constat a été établi pour préciser par petits bassins les superficies irriguées en 1986 par origine de l'eau utilisée ainsi que les mêmes données prévisionnelles pour l'an 2000 (tableau ci-dessous). ture par pompage direct dans les rivières ou dans la nappe alluviale.

Pour ce faire, il a été bien évidemment appliqué sans restriction aux agriculteurs désirant irriguer l'article 410 de la loi 84512 du 29 juin relative à la pêche précisant que « le débit minimal ne doit pas être inférieur au dixième du module du cours d'eau au droit de l'ouvrage ou au débit à l'amont de l'ouvrage si celui-ci est inférieur ".

Ainsi, rivière par rivière, ont été calculés le module, le débit réservé, le débit d'étiage à fréquence quinquennale, donc le débit disponible à l'étiage.

La synthèse par petit bassin de toutes ces données a permis de déduire les volumes à stocker pour faire face aux besoins actuels et à moyen terme de l'irrigation.

L'inventaire exhaustif des retenues de plus de $200000 \mathrm{~m}^{3}$ existantes, établi par l'Agence de Bassin pour les trois régions Midi-Pyrénées, Aquitaine et Poitou-Charente, a répertorié une capacité totale d'environ $300 \mathrm{hm}^{3}$.

L'analyse réalisée a montré alors que c'est un volume de 38 millions de $\mathrm{m}^{3}$ qui devrait être réalisé en première urgence et de $100 \mathrm{hm}^{3}$ environ avant l'an 2000 pour satisfaire les besoins prévisibles de l'agriculture.

\section{Les Contrats de Plan 1989-1993}

Compte tenu de la nécessité d'accroître les ressources en eau disponibles pour l'agriculture dans le Sud-Ouest mais aussi dans les autres régions méridionales, le Ministère de l'Agriculture et de la Forêt a fortement augmenté ses dotations budgétaires affectées à la construction de grands ouvrages : entre 1987 et 1989, les crédits correspondants ont été augmentés de $50 \%$, l'objectif étant de pouvoir participer aux programmes importants prévus dans le cadre des contrats Etat-Régions, conjointement avec les collectivités et les Agences de bassin.

Dans le Sud-Ouest en particulier, la quasi-totalité du programme de moyens et grands ouvrages présenté par les régions pourra être engagé ou réalisé :

- en Poitou-Charente, le Ministère de l'Agriculture participera au financement de quatre grands ouvrages représentant une capacité de près de 70 millions de $\mathrm{m}^{3}$;

- en Aquitaine, le programme engagé porte sur une vingtaine d'ouvrages représentant au total 100 millions de $\mathrm{m}^{3}$; - en Midi-Pyrénées, le programme retenu porte :

1. sur une quinzaine d'ouvrages de capacité comprises entre 1 et $5 \mathrm{Mm}^{3}$ représentant un volume stocké de $40 \mathrm{Mm}^{3}$ environ ;

2. sur la mise à l'étude d'un projet de grand barrage en vue de la réalimentation de la Garonne, dont la réalisation serait engagée après le $\mathrm{X}^{\mathrm{e}}$ Plan.

L'Agriculture n'étant pas le seul utilisateur des réserves ainsi constituées (les grands ouvrages sont très souvent à but multiple), on constate ainsi que le programme lancé permettra globalement de satisfaire les besoins de l'agriculture à la fin du siècle.

Face à cette demande, ont été évalués par sous-bassins les débits et les volumes dont pouvait disposer l'Agricul- 


\section{Conclusion}

La sécheresse des années 1985-1986 a confirmé la faiblesse des débits d'étiage de nombreuses rivières du Sud-Ouest, facteur limitant pour le développement des irrigations.

L'analyse de la situation a conduit à élaborer un programme important de réalisation du renforcement de la ressource en eau, portant sur une capacité d'environ $200 \mathrm{Mm}^{3}$.

Il faut noter qu'une grande partie de cette ressource nouvelle sera créée grâce à des ouvrages de capacité moyenne (entre 1 et $5 \mathrm{Mm}^{3}$ ), permettant ainsi de mener une politique d'aménagement du territoire en diffusant dans de nombreux sous-bassins les ressources ainsi créées.

Cette politique pourra être conduite grâce à un effort financier important de l'ensemble des partenaires (collectivités - Ministère de l'Agriculture - Agences de bassin).

L'agriculture ne sera pas la seule bénéficiaire de cet effort important : globalement, les communes (grâce à l'accroissement des ressources en eau disponibles) et le milieu naturel (grâce à l'augmentation des étiages) bénéficieront aussi de ces réalisations qui permettront à une partie de l'agriculture de ces zones d'être mieux protégés des sécheresses. 